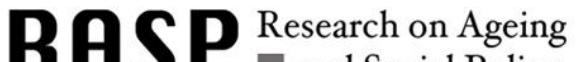
and Social Policy

Instructions for authors, subscriptions and further details:

http://rasp.hipatiapress.com

\title{
Investigación de la Vejez en Pueblos Indígenas de México
}

Laureano Reyes Gómez

1) Universidad Autónoma de Chiapas. México

Date of publication: July $30^{\text {th }}, 2019$

Edition period: July 2019- December 2019

To cite this article: Reyes Gómez, L. (2019). Investigación de la Vejez en Pueblos Indígenas de México. Research on Ageing and Social Policy, 7(2), 339-362. doi: 10.4471/rasp.2019.4292

To link this article: http://dx.doi.org/10.4471/rasp.2019.4292

\section{PLEASE SCROLL DOWN FOR ARTICLE}

The terms and conditions of use are related to the Open Journal System and to Creative Commons Attribution License (CCAL). 


\title{
Investigación de la Vejez en Pueblos Indígenas de México
}

\author{
Laureano Reyes Gómez \\ Universidad Autónoma de
}

Chiapas

(Recibido: 2 mayo 2019; Acceptado: 6 julio 2019; Publicado: 30 julio 2019)

\section{Abstract}

El trabajo busca dar cuenta respecto de la evolución que han tenido los estudios sociales de vejez en pueblos indígenas de México, bajo tres modelos teóricos: el etnográfico, que brinda una visión homogénea de la vejez masculina con rasgos gerontocráticos, explicada en términos culturales. El modelo etnogerontológico se distingue por ser más incluyente, se pueden apreciar diversas formas de envejecer, fenómeno explicado como respuesta a influencias sociodemográficas mundiales. El modelo de antropología de la globalización, se caracteriza por la emergencia de nuevos viejos en un mundo globalizado. La metodología se basa principalmente en análisis cualitativo, auxiliada de información sociodemográfica que permitió clasificar y distinguir cómo se ha estudiado la vejez. Así, las investigaciones de corte etnográfico cubren un período de 1940 a 1999 y se ocupa de estudiar el poder ejercido por los viejos masculinos a través de la gerontocracia; los estudios etnogerontológicos abarcan del año 2000 a 2016, y dan cuenta de la heterogeneidad de la vejez. Las investigaciones de la vejez en mundo globalizado dan inicio en el año 2017, buscan explicar cómo se vive la vejez en un mundo globalizado en situaciones de alta marginación y pobreza, faltos de servicios de atención integral geriátrica y gerontológica.

Keywords: vejez, indígenas, pobreza

2019 Hipatia Press

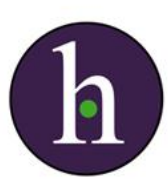

ISSN: 2014-6728

DOI: $10.4471 /$ rasp.2019.4292 


\title{
Research on Old Age in Indigenous Peoples of México
}

\author{
Laureano Reyes Gómez \\ Universidad Autónoma de
}

Chiapas

(Received: 2 May 2019; Accepted: 6 July 2019; Published: 30 July 2019)

\section{Resumen}

The article seeks to account for the evolution of social studies of old age in indigenous peoples of Mexico, under three theoretical models: ethnographic, which provides a homogeneous view of male age with gerontocratic features, explained in cultural terms. The ethnogerontological model is distinguished by being more inclusive, you can see different ways of aging, a phenomenon explained as a response to global sociodemographic influences. The anthropology model of globalization is characterized by the emergence of new elders in a globalized world. The methodology is based mainly on qualitative analysis, aided by sociodemographic information that allowed us to classify and distinguish how old age has been studied. Thus, ethnographic research covers a period from 1940 to 1999 and deals with studying the power exercised by the old men through gerontocracy; Ethnogerontological studies cover the year 2000 to 2016, and account for the heterogeneity of old age. The investigations of old age in a globalized world begin in 2017, seeking to explain how old age lives in a globalized world in situations of high marginalization and poverty, lacking in comprehensive geriatric and gerontological care services.

Palabras clave: old age, indigenous, poverty

2019 Hipatia Press

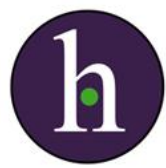

ISSN: 2014-6728

DOI: $10.4471 /$ rasp.2019.4292 


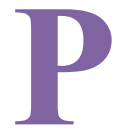

ara el proceso y análisis de la información se recurrió a la metodología cualitativa, con auxilio de información sociodemográfica. Así, se dividió a la población en estudio considerando, básicamente, dos criterios: el modelo teórico de estudio de la vejez en pueblos originarios y la distinción generacional del grupo en estudio, según nacimiento por cuartos de siglo. Así, fueron clasificadas las investigaciones sobre vejez de personas nacidas entre 1900 y 1924, bajo el modelo gerontocrático; la segunda generación de viejos fueron los nacidos en el segundo cuarto de siglo, de 1925 a 1949, y considerados dentro del modelo etnogerontológico. Finalmente, la tercera generación de viejos son los nacidos en el tercer cuarto de siglo, 1950 a 1974, y catalogados dentro del modelo teórico como Antropología de la globalización.

Las investigaciones del primer modelo se caracterizan por explicar la vejez masculina en un mundo idealizado que dibuja un paraíso prácticamente gerontocrático; el etnogerontológico, es más incluyente, y las formas de vivir y atender la vejez se diversifican; en tanto que el tercer modelo, el de la antropología de la globalización, es explicada por la influencia de una economía neoliberal que da lugar a nuevos viejos viviendo en un mundo globalizado.

\section{Introducción}

En las Ciencias Sociales, especialmente en la etnología y la antropología, se han realizado estudios que muestran que los ancianos han sido respetados y venerados en las culturas ancestrales, y el caso mexicano no es la excepción. En los pueblos indígenas de México hay reportes que señalan un trato preferencial hacia los viejos, acompañado de admiración, respeto, obediencia y protección. La vejez era percibida como un período de profundo respeto, toda vez que representaba autoridad moral y liderazgo político-cultural. Ser indígena y conquistar edades avanzadas era la fórmula perfecta para vivir el último tramo del ciclo de vida prácticamente en un paraíso gerontocrático caracterizado por afecto, cuidados y atenciones hasta la muerte. Desde 1940 aparecieron los primeros reportes etnográficos que brindaban información de sociedades gobernadas por sistemas gerontocráticos patriarcales, confiando en los ancianos, por la experiencia y 
sabiduría que los años les daban, el ejercicio de un buen gobierno y el liderazgo para dirigir los destinos de la comunidad. Haciendo un análisis de estos trabajos, el presente apartado busca dar cuenta respecto del desarrollo de los tratados antropológicos realizados en esta materia. Para el seguimiento de las investigaciones, los estudios se clasifican en tres modelos teóricos, divididos según el nacimiento de la población envejecida, por cuarto de siglo, a partir de 1900 a la fecha. Las características generales de cada modelo interpretativo de la ancianidad se sintetizan a continuación.

El primer modelo teórico es identificado como "Etnografía de la vejez". La población anciana investigada en este período nació en el primer cuarto del siglo XX (1900-1924), conformada principalmente por analfabetas y monolingües en lengua nativa. El interés de estos trabajos se centra, básicamente, en estudiar el poder gerontocrático que ejercen los ancianos varones en la comunidad indígena, a través del Consejo de Ancianos y los sistemas normativos indígenas conocidos como "usos y costumbres". El ingreso a la vejez no está del todo definido, y es visto como un fenómeno homogéneo, caracterizado por una ancianidad feliz, sana, lúcida, activa y con ejercicio de poder vertical sobre la aldea; el cuidado y protección brindado a los ancianos es explicado como respuesta cultural que tiene la comunidad hacia los mayores al activarse las redes afectivas y solidarias frente al envejecimiento. El porcentaje de viejos es escaso, oscilaba entre $1 \%$ y $3 \%$ a nivel nacional, y la esperanza de vida se estimaba en 30 años (Peláez, 2009). La mujer anciana, al estar despojada de estatus social alto, pasó prácticamente desapercibida en los reportes etnográficos.

El segundo modelo teórico es llamado "Etnogerontología". La población estudiada en esta etapa son viejos nacidos en el segundo cuarto del siglo XX (1925-1949). Varios rasgos distinguen este modelo del anterior, por ejemplo: hay un boom en los estudios sobre vejez en general. Los nuevos hallazgos en comunidades indígenas reportan que al diversificarse el interés de las investigaciones más allá del ejercicio del poder masculino, la mujer anciana es considerada en los estudios, y la visión reducida que se tenía de la vejez patriarcal es más incluyente no sólo por cuestión de género, sino de temas y problemas de investigación. Por otro lado, el crecimiento de la población de viejos no tiene precedentes en la historia demográfica del país, y se explica como respuesta a un fenómeno 
epidemiológico y demográfico mundial; el porcentaje de adultos mayores se multiplica respecto del modelo anterior al pasar de 3\% a 7.3\% en el año 2000 (Villasana, 2006), y la esperanza de vida se incrementa a más del doble al extenderse de 34 a 74 años (Peláez, 2009). El poder antes ejercido por los viejos es arrebatado por población joven y madura a través de colores partidistas, ahora más bilingües, con algún grado de educación escolarizada y con mayores relaciones con el exterior. La cultura, por sí sola, resulta insuficiente para explicar los cambios que experimenta la comunidad en las formas de percibir y atender el envejecimiento constante de la población, en especial cuando los mayores crean dependencia en cuidados y atenciones y las enfermedades crónico-degenerativas, como las demencias, y otras discapacidades, encuentran huéspedes en la población envejecida.

Las formas de vivir la vejez se diversifican en varias "carreras", y ser viejo e indígena ya no garantizan, de facto, la admiración, respeto, protección, lazos afectivos y solidarios y de veneración que el modelo gerontocrático anterior presumía. El saldo social que motivó el envejecimiento de la población de 60 y más años de edad fue negativo, toda vez que no se tenían previstos los servicios de atención integral a los adultos mayores. El velo que cubría el misticismo de conquistar edades avanzadas se desvaneció, dando paso al registro de los estigmas, la discriminación, el conflicto intergeneracional y los diversos tabúes, como el maltrato al viejo, se hicieron más visibles. En un mundo gobernado por población joven y madura la juventud se ensalza, en tanto que la vejez se estigmatiza. Surgen o se refuerzan síndromes gerascofóbicos y gerontofóbicos que brindan una visión de la vejez como una etapa contraria al modelo etnográfico donde, ahora, ser viejo no necesariamente es la mejor etapa de la vida, y la familia no siempre es el mejor refugio para la persona mayor en etapa dependiente en cuidados y atenciones, especialmente si se convierte en una "carga", por otro lado, se reconoce abiertamente que el viejo también puede tener conductas criminales y perversas, comportamientos que contravienen la idealización de la vejez, y son usados como contraejemplos de la veneración de la edad adulta mayor como modelo a seguir. 
El tercer modelo teórico surge a partir de 2017, y es registrado como "Antropología de la globalización". Los adultos mayores investigados bajo este modelo son conocidos como "nuevos viejos", toda vez que nacieron en la década de los 50 del siglo pasado (1950-1959), y están plenos en todas sus funciones. Estos nuevos viejos presentan características sociodemográficas que los distinguen de los viejos de otras generaciones, por ejemplo: generalmente saber leer y escribir y son, al menos, bilingües en lengua nativa y castellano; son más receptivos al cambio tecnológico, quienes tuvieron trabajo asalariado ahora son jubilados y gozan de pensión, otros más son migrantes de retorno; varios de ellos tienen hijos profesionistas o estudiando en instituciones de educación superior, algunos son profesionistas y viajan, residen en ciudades y/o tienen vivienda en la localidad de origen. El porcentaje de viejos se eleva a $10.4 \%$, y la esperanza de vida es de 75.3 años (CDI, 2016). Los temas de investigación en curso obedecen a las exigencias de un mundo globalizado y de una economía neoliberal, se buscan respuestas a políticas de derechos humanos, diversidad sexual, segundas nupcias, el castigo autoinfligido, abusos y malos tratos, suicidio, demencias, entre otros muchos tópicos. Los niveles de pobreza y alta marginación en las comunidades indígenas y rurales se mantienen. Se hace hincapié en la necesidad de servicios de atención integral geriátrica y gerontológica; la población exige mejores respuestas de los programas de salud. Bajo estos esquemas explicativos damos paso al desarrollo de cada modelo teórico de las investigaciones sociales, que sobre vejez se han hecho en poblaciones indígenas y rurales de México. En forma esquemática se explica este proceso en el cuadro 1.

\section{Cuadro 1.}

Modelos teóricos y características de estudio social de la vejez en pueblos indígenas México.

\begin{tabular}{|l|l|l|l|c|c|}
\hline Modelo teórico & Generación estudiada & Edad al 2018 & $\begin{array}{c}\text { Tema de } \\
\text { investigación }\end{array}$ & $\begin{array}{c}\text { \% de } \\
\text { viejos }\end{array}$ & $\begin{array}{c}\text { Esperanza } \\
\text { de vida }\end{array}$ \\
\hline Etnográfico & $1900,1924,1925,1949$ & 94 el más joven & Gerontocracia & 1 y 3 & 30 \\
\hline $\begin{array}{l}\text { Etnogerontológi } \\
\text { co }\end{array}$ & & 69 el más joven & $\begin{array}{l}\text { Envejecimiento de la } \\
\text { población y } \\
\text { heterogeneidad de la } \\
\text { vejez }\end{array}$ & 3 y & 7.3 \\
\hline $\begin{array}{l}\text { Antropología de } \\
\text { la globalización }\end{array}$ & 1950,1959 & $\begin{array}{l}\text { Nuevos viejos en un } \\
\text { mundo globalizado }\end{array}$ & 10.4 & 75.3 \\
\hline
\end{tabular}

Fuente: Elaboración propia con datos de gabinete. 


\section{El Modelo Etnográfico}

Para hablar de vejez en general, y de vejez en contextos rurales o indígenas en particular, es preciso hacer los distingos correspondientes, y no tratar el asunto como un fenómeno homogéneo y estático; sobre el particular, Bazo (1992, p. 78) opina:
Al hablar de las personas en edades comprendidas entre los cero y los cuarenta años se suelen realizar distinciones según los tramos de edad diferentes en las que se encuentran. ¿Por qué se tiende a homogeneizar a las personas de '65 y más años' con las de 80, 90 o incluso 100 años?

El proceso de envejecimiento está vinculado a dinámicas epidemiológicas, demográficas, tecnológicas, culturales, económicas e históricas, que modifican la estructura poblacional, influyendo en las formas de percibir y atender la edad avanzada; su diversidad nos permite identificar y clasificar las investigaciones por generaciones teóricas; de esta forma fueron construidos nuestros modelos explicativos de vejez aquí expuestos, observando que la vejez, y el proceso de envejecimiento en pueblos indígenas no son tan "tradicionales" como se suponía, o en palabras de Ham (1999, pp. 12-13) "estas experiencias se contraponen a los estereotipos que en lo urbano idealizan a la población anciana en el campo y entre los indígenas".

Así, el primer modelo está construido sobre la idea central en el pensamiento de que "una larga vida es una bendición... una recompensa que obtenía el individuo por el debido acatamiento de los principios comunitarios" (De Lima y Jaber, 2009, p. 14). Se creía que sólo las personas que poseían tonas (tonalli, alter ego) poderosas eran capaces de conquistar edades avanzadas (López-Austin, 1984). El ingreso a la vejez no estaba del todo definido, los criterios etario, biológico, legal y social no se discuten (Envejecimiento productivo, 2015, p. 11), toda vez que

La vejez entre los indígenas no es establecida por ningún ritual o ceremonia de pasaje; es una clase de edad solo percibida, por eso se 
hace difícil identificar, entre la población adulta mayor, quién está al principio o al final del proceso [de] envejecimiento

Considerando que la esperanza de vida a finales del primer cuarto del siglo XX era de 34 años (INEGI, 2016), y el porcentaje de viejos se calculaba entre 1\% y 3\% (Peláez, 2009), la vejez se alcanzaba a edades más tempranas. Era, entonces, tarea del etnógrafo estimar quién era viejo, advirtiendo que "en la mayoría de los pueblos ágrafos los individuos no saben con exactitud su edad cronológica, y generalmente tampoco tienen interés en saberla, ya que en los contextos culturales en los que viven carece de importancia" (Fericgla, 1992, p. 59) ${ }^{1}$. Por otro lado, los pueblos originarios cuentan con sistemas propios para estimar la edad, y la dividen por etapas, que no se ajustan, necesariamente, a sistemas cronobiológicos; los zoques de Chiapas, por ejemplo, asocian el ciclo vital a las diversas fases del sol (Reyes, 2016).

Las investigaciones antropológicas realizadas entre 1940 y 1998 estudiaron principalmente a la generación de viejos nacidos en el primer cuarto de siglo XX (1900-1924), cuyas características dominantes de vejez "tradicional" eran percibidas justamente como un fenómeno homogéneo, donde el viejo masculino, gracias al conocimiento profundo que tenía de la cultura del grupo, lo usaba a su favor, y le permitía ejercer el poder en forma vertical sobre la comunidad. Esta generación inició la vejez a partir del año 1960, y llegó a duplicar la esperanza de vida.

El viejo indígena, en su papel de líder, era sumamente respetado, escuchado, obedecido, temido, procurado y atendido hasta su muerte; la comunidad en su conjunto se hacía cargo de los viejos. Ser anciano bajo las características de veneración era lo máximo, gozaba de alto estatus social; bajo este modelo el conflicto intergeneracional no existe, toda vez que los sistemas normativos indígenas dictan que el poder se ejerce bajo usos y costumbres de la gerontocracia. Este tipo de vejez reverencial llamó la atención de los etnógrafos, centrando el interés en el poder que el viejo ejercía sobre la comunidad indígena y el manejo que tenía de la cultura. El estudio de la vejez era tratado como un tema "exótico", y mostraba la cara "bonita" de la edad avanzada, el de una persona que vive una vejez además de venerable, plena, feliz, sana, lúcida, activa y exitosa. Los viejos, 
entonces, eran tratados a tabla rasa, sin hacer distingos por grupos de edad o sexo.

Los ancianos, además del ejercicio del poder, traducido en "usos y costumbres", eran los depositarios del conocimiento de la cultura del grupo, y formaban su reemplazo en futuros líderes o heredaban el "don" en algún familiar la conducción habilidosa del ritual, de las costumbres, del manejo de la lengua nativa, etcétera; el reconocimiento social de los iniciados lo conquistaban con méritos. Entre otras especialidades era común la formación en artes como la música, danza, artesanía, pintura, escultura; el ejercicio de la medicina como la herbolaria, partería, hueseros; la magia y la hechicería, la lectura de oráculo, agoreros, adivinos, intérprete de sueños, manejo del calendario agrícola, etcétera. En el campo religioso sobresalían los rezadores, los organizadores de los diversos rituales, como los de iniciación o de actos fúnebres, etcétera. Los casamenteros, quienes cultivaban un lenguaje culto aplicado en discursos conocidos como "palabras que vuelan sobre alas de mariposa", pretendían, a través de la elocuencia, ablandar corazones de los celosos padres para autorizar el matrimonio de sus hijas. Los ancianos habilidosos en el manejo del discurso persuasivo eran utilizados también como albaceas, consejeros, mediadores de conflictos y cabildeo. Los ancianos en la comunidad eran los abuelos, los terapeutas, los maestros en diversas áreas del conocimiento, los líderes morales, la autoridad. Como podemos apreciar, los ancianos que lograban dominar habilidades en diversos planos del conocimiento eran considerados sabios, a tal grado que fueron llamados "bibliotecas andantes" (García, 2008, p. 91). Cuando algún joven faltaba el respeto a sus mayores, y si el caso era grave, se turnaba a la asamblea general, donde se determinaba la pena a purgar. Por otro lado, habrá de considerarse que la educación de los hijos se hacía bajo reglas muy estrictas y los castigos eran severos en caso de incumplimiento a las normas dictadas por los padres ${ }^{2}$.

El reconocimiento del poder de los viejos era tal, que Calixta Guiteras (1988, pp. 248-249) registró de su informante de los Altos de Chiapas, comparar a los ancianos como a semidioses al considerar que el "calor" de sus cuerpos, adquirido con la edad, puede ser dañino a terceros si no se le guarda el debido respeto. Sobre el particular refiere: 
lo relaciona con los dioses o seres poderosos que conservan y destruyen la vida. Por medio del "calor" el hombre se asemeja a Dios... Esto significa que tales personas pueden conservar y destruir, defender y devorar; y el ser humano padecerá en caso de que no les guarde el respeto que se les debe.

Otro tanto sucede en la mitología de los zoques de Chiapas en relación a Pyogbachu'we ("la Vieja que Arde"), deidad femenina extremadamente bella, rica y poderosa; se cree vive en el interior del cráter del volcán Chichón. Un ropaje que a menudo usa es la de anciana que viste harapos y anda mendigando sustento, probando la bondad de sus habitantes para con los ancianos desvalidos. Si alguien le niega ayuda podría desatar su furia haciendo erupción el volcán e inundando de fuego y cenizas no sólo las pueblos zoques, sino al mundo entero, tal como sucedió entre el 28 de marzo y el 5 de abril de 1982 cuando tuvo actividad explosiva violenta, expulsando de su territorio a más de 20 mil indígenas zoques, desencadenando, además, miseria, hambre, desolación y muerte (Báez, et al., 1985).

El trato preferencial brindado a la población envejecida era explicado como una distinción cultural que los pueblos indígenas tenían sobre sus mayores expresada a través del respeto irrestricto, por haber logrado éstos conquistar edades avanzadas y acumular conocimientos y experiencia. Además, la persona que llegaba a la vejez, producto de una fuerte selección biológica, era percibida como investida de poderes mágicos en virtud de haber sobrevivido a situaciones adversas de la vida (enfermedades, hambrunas, epidemias, guerras, accidentes, fenómenos naturales, discapacidades, etcétera), y era reconocida por la comunidad como sabia, líder, consejera que manejaba los destinos de la aldea. La persona vieja se convertía, entonces, como el "papá del pueblo"3, el "principal". En varios asentamientos, cuya propiedad de la tierra era comunal, funcionó un órgano de gobierno que se llamó Consejo de Ancianos, desde donde un grupo selecto de viejos, todos varones, ejercía la gerontocracia.

Los sistemas normativos indígenas, como el Consejo de Ancianos, eran foco de atención de la Antropología. Neiburg (1988, pp. 127-128) estudió a los mazatecos de Oaxaca y el poder del anciano en la sociedad indígena, 
registró que en la zona norte de la Sierra Mazateca sí se ejerce la gerontocracia, toda vez que:

... el Consejo de Ancianos posee injerencia sobre todos los problemas de la comunidad, siendo la función más importante a su cargo la elección del presidente municipal y de todas las autoridades que conforman el municipio, como también el control sobre las autoridades para que ejerzan 'un buen gobierno'... la relación que existe entre las autoridades [indígenas] y las formales [oficiales] indica que el verdadero poder de la comunidad está ejercido por los viejos, quienes controlan en todo momento las actividades y las acciones de los presidentes municipales. Los ancianos, a nivel individual, intervienen en todos los planos de la vida de la comunidad, en los casamientos, en los bautizos, en todos los ritos relacionados con las alianzas entre las familias.

Respecto de la vejez femenina conocemos muy poco, en virtud de que los estudios se realizaron sobre la autoridad masculina, donde la mujer aparece subyugada al poder patriarcal. En 1944 sale a la luz un trabajo pionero donde se hace referencia a la vida de la mujer tzeltal de Oxchuc, Chiapas, cuando alcanza la ancianidad. Sobre el particular Lombardo (1944, pp. 57-58) reporta:

... en la casi totalidad de los casos, la mujer no posee nada. Esto trae como consecuencia social, que la mujer, en muchas ocasiones, se convierta en un ser sin casa fija, teniendo que trabajar con alguna familia, para que le den en pago un poco de pozole para comer y un rinconcito en la humilde choza para pasar la noche y protegerse un poco del intenso frío que hay en la montaña

Estas mujeres desheredadas, ya por haber quedado viudas y sin hijos varones o por ser estériles, tienen, además de sufrir las humillaciones que hemos indicado, que vestirse con harapos que les regalan, pues nunca pueden comprar un vestido nuevo por carecer de dinero.

La vida de la mujer en la vejez está todavía más llena de sufrimientos, pues vive única y exclusivamente de la compasión de los miembros de la comunidad, debido a que por su edad ya no puede desempeñar casi ningún 
trabajo con el cual pagar su alimento y el rincón de una choza donde dormir".

A menos que la mujer dominara habilidades terapéuticas como el ejercicio de la partería, lograba conquistar un estatus social alto, y era sumamente respetada. En lengua zoque la comadrona es conocida con el término reverencial de oko y es considerada dentro la relación de parentesco ritual como abuela ${ }^{4}$. Otro tanto sucede con las artesanas, rezadoras, danzantes, cuentacuentos, sobadoras, yerberas, levantadoras de espanto, entre otras especialidades, que cuentan con el respeto y apoyo moral de la comunidad.

Con los elementos hasta acá expuestos nos permite entender que el trato preferencial que se aduce gozaban los ancianos varones en este primer modelo explicativo, está fundado en varios soportes, a saber:

a) La escasez de ancianos y la baja esperanza de vida hacía de los longevos una élite, reconocida por su experiencia y sabiduría que los años les daban. La ancianidad, entonces, estaba cubierta por un velo de misticismo que permitía reconocer en el anciano funciones de liderazgo. El viejo infundía respeto, temor y obediencia; se recurría a ellos en su papel de consejero, y era común besarle el dorso de la mano solicitando su bendición.

b) La formación de los hijos se hacía bajo reglas muy estrictas; se inculcaba el respeto y soporte a sus mayores con muestras de afecto y solidaridad.

c) El anciano se valía del sistema normativo indígena para ejercer la gerontocracia. El joven tenía que acatar todas las disposiciones de sus mayores, so pena de sufrir castigos con penas muy severas.

d) Se reconoce al anciano como la única fuente de conocimientos en materia de cultura indígena del grupo; son los expertos en varias áreas del conocimiento como en la medicina, el arte, la magia y la hechicería. Los viejos son los maestros formadores de generaciones de reemplazo.

e) El sistema de gobierno es patriarcal; la mujer no tiene mayor injerencia en la vida política de la comunidad, su opinión no es considerada en la toma de decisiones; la autoridad de los viejos los investía para hacer arreglos matrimoniales. 
f) La vejez es percibida como un periodo donde la vida está llena de cuidados, atenciones, protección y soporte por parte de los suyos y de la comunidad en su conjunto.

g) La vejez es descrita en los estudios gozando el mejor período de la vida, siendo el viejo venerado, pleno, lúcido, activo, feliz y exitoso. Ser viejo bajo estos considerandos era lo máximo, es la retribución por haber sobrevivido a las vicisitudes de la vida.

h) La figura del viejo en la comunidad era un ejemplo a seguir por su bonhomía, sabiduría y liderazgo.

\section{El Modelo Etnogerontológico}

Los viejos estudiados bajo este modelo nacieron principalmente en el segundo cuarto del siglo XX, entre 1925 y 1949; esta generación inició la vejez a partir de 1985. Para entonces, el país experimentaba cambios socioculturales muy importantes, por ejemplo, en el terreno demográfico el Programa Nacional de Población 1995-2000 (Poder ejecutivo federal, 2000), advertía que el crecimiento de la población adulta mayor (65 y más años de edad) era producto de la transición demográfica mundial, y tal fenómeno no tenía precedentes. Sobre el particular, apunta:

El grupo de población de 65 años y más representa hoy día sólo el 4 por ciento de la población -con 3.7 millones de personas-, pero su crecimiento es muy marcado: pasó de menos de 1.0 por ciento anual en 1960 al 4.0 por ciento en 1990, y se ha mantenido en este nivel desde entonces. Mientras en 1960 los incrementos anuales eran de menos de 20 mil individuos, actualmente ascienden a $150 \mathrm{mil}$. La dinámica de crecimiento de este grupo refleja la rapidez del proceso de envejecimiento de la población mexicana: la tasa de crecimiento que desde 1990 alcanzó la población de la tercera edad es inédita en la historia demográfica del país, y se estima que se mantendrá en ese orden de magnitud en el mediano plazo.

Hay un boom en estudiar explícitamente las formas de concebir, entender y explicar la vejez y el desarrollo del envejecimiento en México. La academia muestra preocupación por la transición demográfica y 
epidemiológica, y para su análisis celebra seminarios, congresos, publicaciones, conferencias, documentales. La ONU, la OMS, universidades, institutos y otros organismos públicos crean programas multidisciplinarios de investigación y docencia en vejez y envejecimiento desde varias perspectivas tanto teóricas como metodológicas.

Para conocer explícitamente qué sucede en las poblaciones indígenas y rurales respecto de la vejez, surge una línea de investigación llamada Etnogerontología, cuya disciplina considera el análisis integral de las particularidades socioculturales de la población de edades avanzadas en grupos étnicos de México. La preocupación por conocer el envejecimiento de poblaciones rurales e indígenas está sustentada por los altos índices de marginación, asociadas a pobreza y la respuesta cultural del grupo frente a los mayores. Se partía de la hipótesis que los pueblos originarios, producto de la cultura del grupo y la importancia institucional de los ancianos en la vida comunitaria, la vejez tenía un toque distintivo que la caracterizaba por brindar un trato de respeto y soporte a través de las redes de apoyo solidario y afectivo a la población envejecida (Reyes, 2002).

Así, por criterios legales, demográficos, y conciliando la perspectiva indígena de cuándo se es viejo en la cultura del grupo, se estimó el inicio de la vejez a partir de los 60 años de edad. En el segundo cuarto del siglo XX el porcentaje de viejos indígenas pasó de 3\% a 7.3\% (Villasana, 2006), y la esperanza de vida se incrementó a más del doble, al elevarse de 34 a 75 años (Peláez, 2009). El envejecimiento constante de la población motivó un cambio paradigmático en las formas de percibir y atender la vejez en varios planos. Por ejemplo, la comunidad ya no se podía hacer cargo de los viejos, en especial lo referente a atención en servicios de salud, convirtiéndose muchas veces en una "carga" cuando creaba dependencia y requería, por ejemplo, de servicios hospitalarios especializados. En casos extremos la comunidad no dejaba morir a los ancianos de hambre, especialmente en condiciones de abandono, discapacidad, accidente o viudez; las redes solidarias entran en juego y dan soporte a los más necesitados.

Los niveles de pobreza y alta marginación de las comunidades se agudizaron; la falta de servicios integrales a la población envejecida era prácticamente nula. En el plano jurídico la Federación publica, en el 2002, la Ley de los Derechos de las Personas Adultas Mayores, el cual busca 
brindar protección y "garantizar el ejercicio de los derechos de las personas adultas mayores" (INAPAM, 2002), y convertir al anciano como sujeto de derecho.

De acuerdo al Boletín del Programa Iberoamericano de Cooperación sobre Adultos Mayores (Envejecimiento productivo, 2015, p. 7) en el plano internacional la vejez es considerada como

una de las etapas de la vida, quizá, la más vulnerable; se incluye en este concepto a la población indígena. Las personas adultas mayores cursan por situaciones distintas al resto de la población, esto como consecuencia de los fenómenos demográficos, epidemiológicos, sociales, económicos y políticos por lo que se atraviesa en el país, las personas indígenas no escapan a estas situaciones, debiendo considerar además su contexto cultural.

Aunque existe una tendencia en seguir estudiando el poder de los viejos, los temas se diversifican, considerando a la mujer como actor social. Los nuevos trabajos más bien centran su atención en investigar cómo se dio el desmoronamiento de la estructura de gobierno indígena "tradicional" conocido como Consejo de Ancianos, al competir con otros sistemas y órganos de gobierno como los partidos políticos, la asamblea como máximo órgano de gobierno bajo el sistema de propiedad ejidal de la tierra $y$, en menor medida, el peonaje en las fincas, donde el trabajador era prácticamente un esclavo, sin posibilidad de autogobierno.

La ruptura del sistema gerontocrático se inicia entre 1929 y 1936, cuando el Partido Revolucionario Institucional hace presencia en las comunidades indígenas e impone nuevo sistema de gobierno partidista (Fábregas, 1991, p. 297), dando al traste las formas de gobierno indígena, donde el nuevo sistema de gobierno "desplaza también a los ancianos y otorga preeminencia a los partidos políticos, particularmente (aunque no sólo) al PRI"'5.

Comunidades que aún se regían bajo el sistema de Consejo de Ancianos cambiaron su estructura de gobierno, pasando el Consejo, en el mejor de los casos, como un órgano de consulta, más no de decisiones. Los ancianos "principales" fueron relegados a ejecutar actos rituales donde no ponían en riesgo los acuerdos tomados desde el cabildo (Vázquez, 2006), y los viejos 
que manejaban habilidades en diferentes áreas del conocimiento actuaban a título personal. Con este desplazamiento que sufrió la población anciana ahora el prestigio social, el respeto, el soporte, las redes afectivas y solidarias frente al envejecimiento se conquistaban día a día, y no se ganaban en automático por ser viejo, como presumía el modelo anterior. La autoridad de los viejos sufrió un cambio drástico al pasar de figura de gobierno político y cultural, a líder moral.

El envejecimiento de la población era explicado como respuesta a un fenómeno demográfico y epidemiológico mundial, y el velo de misticismo que cubría la ancianidad se fue desvaneciendo, ahora era cada vez más fácil conquistar edades avanzadas. La mayoría de los viejos, principalmente mujeres, eran analfabetos y monolingües en lengua originaria.

Una vez arrebatado el poder a los viejos el conflicto intergeneracional se agudizó, y las formas de vivir la vejez se diversificaron. La vejez, ahora heterogénea, permitió distinguir tanto a hombres como a mujeres por grupos de edad y sexo, dividiéndolos entre media vejez, vejez completa funcional y vejez extrema dependiente (Reyes, 2002, 2016). La salud del anciano tanto física como mental y la discapacidad, así como las redes de apoyo frente al envejecimiento fueron elementos a considerar para distinguir dependencia, cuidados y atenciones. El envejecimiento constante de la población, el arrebato del poder a los ancianos, la migración, la falta de servicios médicos y asistenciales, altos niveles de marginación y pobreza, así como los procesos de modernización menoscabaron la competencia de los ancianos y dejaron de ser la única fuente de conocimientos, etcétera.

En un mundo gobernado por jóvenes la juventud se ensalza en tanto que la vejez se estigmatiza, y se hizo visible el abuso y los malos tratos a los viejos. En la nueva estructura de vejez heterogénea el viejo ejerce autoridad en la familia y tiene la probabilidad de ser obedecido en tanto se mantenga lúcido, medianamente sano y ejerza el control sobre los bienes medios de producción, y sus redes afectivas sean sólidas. Una vez que el viejo sufre demencia en grado avanzado podría ser su ruina, pues ya no es considerado en la toma de decisiones, por el contrario, el anciano deja de ser "alguien", y corre el riesgo de ser tratado con desdén. Investigaciones recientes señalan que la esperanza de vida sana al nacer es de apenas 65 
años. Gutiérrez y Caro (2013) dicen que el verdadero reto de hoy en día no es prolongar la vida, sino mantener la salud al envejecer.

Los temas de investigación sobre vejez en comunidades indígenas y rurales se diversifican, y muestra las otras caras de la vejez, que en el modelo anterior podrían ser contraejemplos de la visión gerontocrática. Así, en Veracruz surgen estudios pioneros sobre religión, muerte, pescadores y cambio climático (Vázquez, 2003, 2006, 2009). En Chiapas, Villasana (2006) analiza el envejecimiento de la población indígena de México en situaciones de pobreza extrema y alta marginación. Reyes $(2002,2016)$ en Chiapas, estudia el significado social de ser viejo en contextos indígenas. Pelcastre (2011) en Chihuahua, Morelos y Yucatán investiga la salud de los viejos en situaciones interculturales. Villagómez $(2013$, 2017) y Villagómez y Vera (2013) se ocupan de la marginación social que sufre la mujer maya en la vejez. Ronzón, Vázquez y Munguía (2017), coordinan el estudio de análisis de la vulnerabilidad en la vejez. Ruelas, Pelcastre y Reyes (2014) y Ruelas, Pelcastre, Alcalde, Ortega, Ruano y Hernández (2017), en Morelos, diseñan una escala geriátrica para detectar el maltrato en instituciones hospitalarias. Aguilar, Díaz y Vázquez (2013), en Quintana Roo, estudian los programas de atención a adultos mayores indígenas mayas de zonas marginadas de Cancún.

Vázquez (2004), pionero en los estudios de vejez en contextos rurales, advierte: "La vejez es el período más largo de la vida", y debemos preparar las condiciones necesarias para ofrecer respuestas apropiadas. Se han dado los primeros pasos en investigación, pero es preciso profundizar en temas tales como violencia, mendicidad, suicidio, maltrato, discriminación, abandono, estigmatización, discapacidad, etcétera, que han sido tratados en forma superficial.

\section{El Modelo de Antropología de la Globalización}

La globalización afectó a los pueblos indígenas, y la población envejecida no fue ajena a los efectos modernizadores que el mundo estaba experimentando. A estos nuevos viejos les tocó vivir los últimos 25 años de cambios tecnológicos acelerados, por lo que son más receptivos al uso de medios electrónicos como computadoras y periféricos, telefonía móvil, 
televisión con señal abierta y satelital, tarjetas de débito, etcétera. Cada vez va siendo común que los viejos que viven en un mundo globalizado usen medios de comunicación electrónicos, y sean usuarios de redes sociales, correo electrónico y mensajería instantánea como Whats App, entre otras aplicaciones; la vejez va acompañada a los efectos modernizadores que como sociedad experimentamos.

En el campo laboral, los que tuvieron trabajo asalariado son ahora jubilados, otros más son migrantes de retorno. Varios de ellos estudiaron una profesión y/o tienen hijos profesionistas o estudiando en instituciones de educación superior. Es común que algunos nuevos viejos residan en ciudades o cuenten con vivienda en la localidad de origen; generalmente viajan al interior de la república, o bien, al extranjero.

La generación de los nuevos viejos tiene una estructura familiar y de residencia más compleja que los modelos anteriores, la migración tanto nacional como internacional permitió que el trabajo se diversificara, por lo que no todos, necesariamente, se dedican a actividades agropecuarias como actividad principal, ya que tuvieron otras opciones laborales. Así, hombres y mujeres, los que estudiaron alguna carrera o fueron contratados por alguna empresa fueron asalariados, otros, pusieron algún negocio propio. Otro más, se auto-emplearon generalmente como choferes, albañiles, carpinteros, jardineros, entre otras actividades.

Esta generación de nuevos viejos nació en la década de 1950 e inició la vejez a partir del año 2010, el más joven de ellos cuenta, hoy en día (2018), con 59 años, es decir, aún no han alcanzado la esperanza de vida, estimada en 75.3 años, con una población de $10.5 \%$ de viejos, igualando a la población mexicana (INEGI, 2017). Generalmente saben leer y escribir, y son, al menos, bilingües en lengua castellana e idioma materno. Por un lado, este tipo de vejez apenas está en su fase inicial, y requiere ser estudiada desde una perspectiva particular, que contemple lo global en una economía neoliberal, por otro lado, la generación de nuevos viejos, por cuestiones de edad, podría presumirse que están plenos en funciones, relativamente sanos, y muchos de ellos se resisten a reconocerse como viejos.

Los niveles de pobreza y marginación se mantienen, la falta de programas de asistencia social y atención médica especializada son escasos o nulos. En épocas electorales el sector envejecido es utilizado como 
bandera política, toda vez que constituye voto clientelar. El impacto de lo global en las comunidades indígenas y rurales en relación a temas de investigación de la vejez refleja intereses que requieren ser explicados de acuerdo a la realidad contemporánea. Áreas como derechos humanos, diversidad sexual, castigo autoinfligido, abusos y malos tratos, suicidio, cuidados y atenciones, demencias, etcétera, son temas emergentes, cuyo estudio debe ser investigado por grupos multidisciplinarios y trabajado en red. Se están haciendo los primeros esfuerzos académicos. En relación a vejez en pueblos afrodescendientes en México no sabemos nada, es un campo del conocimiento aún inexplorado; es preciso hacer los primeros acercamientos que nos dé cuenta de cómo viven la vejez estos pueblos.

Las generaciones egresadas de las carreras de Gerontología tienen un campo fértil de acción, y se espera un cambio paradigmático en beneficio de la población envejecida, otro tanto sucede en la necesidad de impulsar la formación de recursos humanos en servicios integrales para la atención de las personas adultas mayores, considerando las necesidades y la cultura del pueblo indígena. El cumplimiento de las leyes que brindan protección legal a las personas mayores es una exigencia que debe ser cumplida a cabalidad. En materia de investigación gerontológica es preciso redoblar esfuerzos y trabajar en grupos multidisciplinarios que den respuesta a las necesidades y exigencias de los pueblos originarios como acto de justicia social.

\section{Conclusiones}

El estudio de la vejez y el proceso de envejecimiento en México adquiere mayor relevancia cada vez. Existe preocupación en la academia por analizar y explicar cómo se encuentran interrelacionados y su incidencia en el proceso de envejecimiento varios fenómenos, en un país que se declara pluricultural y multilingüe. Por otro lado, organismos internacionales como los derechos humanos y políticas gubernamentales, buscan que las brechas entre los sectores menos favorecidos sean más estrechas. Para lograr mejores condiciones de la población envejecida es preciso hacer más investigación en materia gerontológica, y formar recursos humanos que permita la atención integral al sector envejecido. Se han dado los primeros pasos, pero es preciso reforzarlos. 


\section{Notas}

${ }^{1}$ Don Daniel, indígena zoque de Chiapas, cuando se le preguntó la edad contestó: "No lo sé con exactitud, pero calcule: Me casé a los 20 años de edad, y desde entonces he gastado 8 machetes, y estoy ocupando el noveno. Cada machete dura entre 6 y 8 años".

${ }^{2}$ Era común que a los barones se les hincara con el dorso descubierto y recibieran golpes con reata previamente remojada en agua de sal hasta reventar la piel; las mujeres solían quemarles la boca con el batidor del atole hirviendo.

${ }^{3}$ En lengua nativa zoque existe el término, y es conocido como kubguy jyara (kubguy= pueblo, $j=$ marcado de posesivo de tercera persona en singular, y jara= papá); es decir, "papá del pueblo". En idioma mixteco, por ejemplo, se conoce como "tata mandoñis" (padre del pueblo); en lengua mixe, entre otras designaciones es conocido como "mëj jä'äy" (gente mayor, gran señor). Gracias a la comunicación personal de Antonio Gómez Hernández se sabe que en idioma tojolab'al el viejo "principal" es conocido como olamal (la cabeza, el que encabeza, líder).

4 Villasana (1988, p. 104), al clasificar la tecnonimia de parentesco entre los zoques de Tapalapa, ubica a la partera (okonana) como pseudoparentesco, pues entre la partera y el recién nacido se establece una relación figurada, más no necesariamente de descendencia o de alianza. Traduce okonana como "madre última", proveniente de los lexemas oko= lo que está a lo último, el extremo opuesto a EGO; nana=madre.

${ }^{5}$ Fábregas ubica el cambio de estructuras de poder entre 1929 y 1936, haciéndola coincidir con la fundación del Partido Nacional Revolucionario (PNR) el 4 de marzo de 1929; el 30 de marzo de 1938, el PNR se transforma en Partido de la Revolución Mexicana (PRM), finalmente, el 18 de enero de 1946, adquirió su actual denominación: Partido Revolucionario Institucional (PRI).

\section{Referencias}

Aguilar, P., Díaz, L., y Vázquez, G. (2013). Retos en la atención a los adultos mayores en Cancún, Quintana Roo. In Gina V. \& Ligia V. (Eds.), Vejez. Una perspectiva sociocultural (pp. 185-206). Universidad Autónoma de Yucatán, Universidad Autónoma de Campeche.

Báez, F., Rivera, A., y Arrieta, P. (1985). Cuando ardió el cielo y se quemó la tierra. México: Instituto Nacional Indigenista.

Bazo, T. (1992). La nueva sociología de la vejez. REIS 60(92), 19-31. 
De Lima, B. y Jaber, J. (2009). La mirada inocente: una reflexión sobre el estudio de la gerontología en Venezuela. Altepepaktli. Salud de la Comundad, 10(5), 11-18.

Envejecimiento productivo (2015). Boletín del Programa Iberoamericano de Cooperación sobre Adultos Mayores, 08, p. 11.

Fábregas, A. (1992). Una interpretación de la estructura de poder entre los zoques. In Antropología Mesoamericana. Homenaje a Alfonso Villa Rojas (pp. 289-297). Tuxtla Gutiérrez: Consejo Estatal de Fomento a la Investigación y Difusión de la Cultura.

Fericgla, J. (1992). Envejecer: Una antropología de la ancianidad. Barcelona: Anthropos.

García, M. (2008). Escritoras bolivianas de hoy. Bolivia: La Hoguera.

Guiteras, C. (1988). Los peligros del alma. Visión del mundo de un tzotzil. Cuba: Ciencias Sociales.

Gutiérrez, L. y Caro, E. (2013). "México en el umbral del envejecimiento". Este país, 1 de octubre de 2013.

Ham, R. (1999). El envejecimiento en México: de los conceptos a las necesidades. Papeles de Población, 5(19), 7-21. Disponible en http://www.redalyc.org/pdf/112/11201902.pdf

CDI (2016). Comisión Nacional para el Desarrollo de los Pueblos Indígenas de México. Disponible en https://www.gob.mx/cdi/articulos/indicadores-sobre-adultosmayores-indigenas-de-mexico [consultado el 11 de julio de 2018].

INEGI (2016). "Cuéntame". Disponible en http://cuentame.inegi.org.mx/poblacion/esperanza.aspx?tema $=\mathrm{P}$ [consultado el 20 de junio de 2018].

INEGI (2017). Estadísticas a propósito del Día Internacional de las Personas de Edad. México. Disponible en http://www.inegi.org.mx/saladeprensa/aproposito/2017/edad2017_Na 1.pdf [28 de septiembre de 2017]

INAPAM (2002). Ley de los Derechos de las Personas Adultas Mayores. México: Gobierno Federal.

Lombardo, R. (1940). La mujer tzeltal. México: s/e.

López, A. (1984). Cuerpo humano e ideología. México: Universidad Nacional Autónoma de México. 
Neiburg, F. (1988). Identidad y conflicto en la sierra mazateca: el caso del consejo de ancianos de San José Tenango. México: Ediciones Cuicuilco

Peláez, O. (2009). Descripción y proyección de la esperanza de vida al nacimiento en México. Estudios demográficos y urbanos, 2(71). Disponible en http://www.redalyc.org/pdf/312/31221534008.pdf

Pelcastre, B. (2011). Modelo de atención a la salud para adultos mayores en zonas indígenas. México: Instituto Nacional de Salud Pública.

Poder ejecutivo federal (2000). Programa Nacional de Población 19952000. México: Poder Ejecutivo Federal.

Reyes, L. (2002). Envejecer en Chiapas. Etnogerontología zoque. México: Programa de Investigaciones Multidisciplinarias sobre Mesoamérica y el Sureste-UNAM, Instituto de Estudios Indígenas-Universidad Autónoma de Chiapas.

Reyes, L. (2016). Etnogerontología. La ancianidad en poblaciones zoque de Chiapas. Disponible en: http://www.geriatria.salud.gob.mx/descargas/publicaciones/etnogero ntologia.pdf [consultado el 27 de julio de 2018].

Ronzón, Z., Vázquez, F., y Munguía, V. (2017). Vejez y vulnerabilidad. México: Universidad Autónoma del Estado de México, Gedisa.

Ruelas, G., Blanca, P., y Reyes, H. (2014). Maltrato institucional al adulto mayor; percepciones del prestador de servicios de salud y de los ancianos. Salud Pública de México, 56(6), 631-636. Disponible en http://www.redalyc.org/pdf/106/10632793008.pdf

Ruelas, G., Blanca, P., Monterrubio, E., Alcalde, J., Ortega, D., Ruano, A., y Hernández, P. (2017). Development and validation of a Screening Questionnaire of Family Mistreatment against Older Adults for use in primary care settings in Mexico. Health and Social Care in the Community, 26(1), 102-112. doi: https://doi.org/10.1111/hsc.12466

Valdéz, S. y Pérez, E. (2015). El patrimonio de las personas adultas mayores indigenas. Boletín del Programa Iberoamericano de Cooperación sobre Adultos Mayores, núm. 8. Disponible en: http://www.oiss.org/IMG/pdf/OISS08-DEF.pdf

Vázquez, F. (Ed.) (2003). Contando nuestros días. México: CIESAS. 
Vázquez, F. (2004). Historia de gente grande [CD ROM]. México: CIESAS.

Vázquez, F. (2006). Estatus social y religiosidad en la vejez rural y urbana en México. In Anuario de Estudios Indígenas XI. (pp. 313-327).

Chiapas: Universidad Autónoma de Chiapas.

Vázquez, F. (2009). Construyendo la vida a partir de la muerte. Veracruz: Gobierno del Estado de Veracruz.

Villagómez, G. y Ligia, V. (Ed.) (2013). Vejez. Una perspectiva sociocultural. Yucatán: Universidad Autónoma de Yucatán, Universidad Autónoma de Campeche.

Villagómez, G. (Ed.) (2013). Género y Vejez en México. México: Senado de la República, Universidad Autónoma de Yucatán.

Villasana, S. (1988). Organización social de los zoques de Tapalapa, Chiapas: un análisis de la identidad sociocultural. Estudios Recientes del Área Zoque. Chiapas: Centro de Estudios Indígenas, Universidad Autónoma de Chiapas.

Villasana, S. (2006). Diagnóstico sociodemográfico de los adultos mayores indígenas de México. México: Comisión Nacional para el Desarrollo de los Pueblos Indígenas de México, Programa de las Naciones Unidas para el Desarrollo.

Laureano Reyes Gómez. Doctor en Ciencias Sociales con Especialidad en Estudios Regionales. Investigador en la Universidad Autónoma de Chiapas.

reylau2001@hotmail.com 